\title{
Mixed and Hybrid Finite Element Methods for Convection-Diffusion Problems and Their Relationships with Finite Volume: The Multi-Dimensional Case
}

\author{
Michel Fortin ${ }^{1} \&$ Abdellatif Serghini Mounim ${ }^{2}$ \\ ${ }^{1}$ Département de Mathématiques et Statistique, Université Laval, Québec, G1K 7P4, Canada \\ ${ }^{2}$ Department of Mathematics and Computer Science, Laurentian University, Sudbury, Ontario, P3E 2C6, Canada \\ Correspondence: Abdellatif Serghini Mounim, Department of Mathematics and Computer Science, Laurentian University, \\ Sudbury, Ontario, P3E 2C6, Canada. E-mail: aserghini@cs.laurentian.ca
}

Received: October 30, 2016 Accepted: December 5, 2016 Online Published: January 9, 2017

doi:10.5539/jmr.v9n1p68

URL: http://dx.doi.org/10.5539/jmr.v9n1p68

\begin{abstract}
We introduced in (Fortin \& Serghini Mounim, 2005) a new method which allows us to extend the connection between the finite volume and dual mixed hybrid (DMH) methods to advection-diffusion problems in the one-dimensional case. In the present work we propose to extend the results of (Fortin \& Serghini Mounim, 2005) to multidimensional hyperbolic and parabolic problems. The numerical approximation is achieved using the Raviart-Thomas (Raviart \& Thomas, 1977) finite elements of lowest degree on triangular or rectangular partitions. We show the link with numerous finite volume schemes by use of appropriate numerical integrations. This will permit a better understanding of these finite volume schemes and the large number of DMH results available could carry out their analysis in a unified fashion. Furthermore, a stabilized method is proposed. We end with some discussion on possible extensions of our schemes.
\end{abstract}

Keywords: convection-diffusion, dual mixed and hybrid finite elements, finite volume, upwinding

\section{Introduction}

The aim of this paper is to extend to multidimensional hyperbolic and parabolic problems the results of (Fortin \& Serghini Mounim, 2005). In (Fortin \& Serghini Mounim, 2005) we presented in a mixed finite element framework, two nonstandard formulations in one space dimension allowing, among other things, to recover in a unique fashion, numerous finite volume schemes. We shall present in this paper a two-dimensional extension. To achieve the discretization we shall use mixed finite elements to approximate the convective and diffusive fluxes. We concentrate on the case of the lowest order Raviart-Thomas spaces (Raviart \& Thomas, 1977), though many of our results will be more general. In particular, to obtain higher-order accuracy finite volume schemes less sensitive to partitions of domain, we can follow the same procedure but utilize others usual mixed finite element approximation subspaces of $H(\operatorname{div}, \Omega)$ such as BDM spaces (Brezzi, Douglas \& Marini, 1986).

This paper is organized as follows. In the next section, we systematically build the extension of the formulations of (Fortin \& Serghini Mounim, 2005) to the multi-dimensional case. Results of existence and uniqueness are presented. In Section 3 , we build the numerical schemes on triangular or rectangular partitions of the domain $\Omega$ using the Raviart-Thomas finite elements of lowest degree (Rarviart \& Thomas, 1977) to approximate the fluxes. In Section 4, we show the link between the finite volume methods and our formulations using the suitable quadrature formulas suggested in (Baranger, Maitre, \& Oudin, 1996) (triangular case) or trapezoidal quadrature (rectangular case) to diagonalize the element mass matrix and numerical integration formulas to approximate the term $\int_{K} f(u) \cdot q d x$ (here $\underline{f}(u)$ is the convective flux). Moreover, we focus our attention to the multidimensional extension of some recent schemes. Finally, in Section 5 we discuss how we can also establish the extension of the second method to both convection-diffusion equations and system of equations. We end with some discussion on possible extensions of our schemes. The DMH formulation requires the solution of a linear system; the approach adopted here was suggested by Arnold and Brezzi (Arnold \& Brezzi, 1985). In their method, one eliminates the velocity and the flux unknowns to leave a system for the Lagrange multipliers alone. The lowest order Raviart-Thomas spaces have one Lagrange multiplier unknown per edge.

\section{Multidimensional Extension}

\subsection{Preliminaries}

In this section, we start by presenting a few properties relative to the decomposition of $\Omega$. The proofs can be found 
in Thomas (Thomas, 1977) or Brezzi-Fortin (Brezzi \& Fortin, 1991). Let us consider the following partial differential equation for $u$ :

$$
\frac{\partial u}{\partial t}+\operatorname{div}(\underline{a} u)-\operatorname{div}(v \operatorname{grad} u)=f \quad \text { in } \Omega,
$$

with given initial data $u(x, 0)=u_{0}(x)$ in $\Omega$, and corresponding suitable boundary conditions on $\Gamma=\partial \Omega$. Here the velocity field and the diffusion coefficient (which is positive) are denoted by $a(x, t)$ and $v$ respectively. Moreover, for simplicity and without loss of generality, we restrict our attention to the case where $\operatorname{div}(\underline{a})=0$; taking into account $\underline{a} . \underline{n}=0$ on $\Gamma \times] 0, T\left[\right.$, and assuming that $\underline{a}(., t) \in L^{\infty}(\Omega)$; also, that $u=0$ on $\left.\Gamma \times\right] 0, T[$.

Let then $T_{h}=\left\{K_{i}\right\}_{i=1}^{N T}$ be the usual non-overlapping finite element triangulation of the domain $\Omega=\cup_{K \in T_{h}} K$. We also consider for $1 \leq t<\infty$ the spaces

$$
X^{t}(\Omega)=\left\{\underline{v} \in\left(L^{t}(\Omega)\right)^{2},\left.\underline{v}\right|_{K} \in W_{t}(\operatorname{div} ; K) \quad \forall K \in T_{h}\right\},
$$

and

$$
W_{t}(\operatorname{div} ; \Omega)=\left\{\underline{v} \in\left(L^{t}(\Omega)\right)^{n} ; \quad \operatorname{div}(\underline{v}) \in L^{t}(\Omega)\right\},
$$

which are both Banach spaces endowed with the usual norms

$$
\|\underline{v}\|_{X^{t}(\Omega)}=\|\underline{v}\|_{0, t, \Omega}+\sum_{K \in T_{h}}\|\operatorname{div}(\underline{v})\|_{0, t, K},
$$

and

$$
\|\underline{v}\|_{W_{t}(\operatorname{div} ; \Omega)}=\|\underline{v}\|_{0, t, \Omega}+\|\operatorname{div}(\underline{v})\|_{0, t, \Omega} .
$$

The following proposition, showing that the space $W_{t}(\operatorname{div} ; \Omega)$ can be characterized as a subspace of $X^{t}(\Omega)$, is well known:

Proposition 1 Let $s>1$, a vector $\underline{q} \in X^{t}(\Omega)$ then $\underline{q} \in W_{t}(\operatorname{div} ; \Omega)$ if and only if

$$
\sum_{K \in T_{h}}\langle\underline{q} \cdot \underline{n}, v\rangle_{W^{-1 / t, t}(\partial K) \times W^{1 / t, s}(\partial K)}=0,
$$

for all $v \in W_{0}^{1, s}(\Omega)$, where $t$ is the conjugate of $s$.

Finally, let us recall that for all $\mu \in W^{1 / t, s}(\Gamma)$ and $\mu^{*} \in W^{-1 / t, t}(\Gamma)$ we have

$$
\|\mu\|_{1 / t, s, \Gamma}=\sup _{\underline{q} \in W_{t}(\operatorname{div} ; \Omega)} \frac{\langle\underline{q} \cdot \underline{n}, \mu\rangle_{\Gamma}}{\|\underline{q}\|_{W_{t}(\operatorname{div} ; \Omega)}},
$$

and

$$
\left\|\mu^{*}\right\|_{-1 / t, t, \Gamma}=\sup _{v \in W^{1, s}(\Omega)} \frac{\left\langle\mu^{*}, v\right\rangle_{\Gamma}}{\|v\|_{1, s, \Omega}} .
$$

Here $\langle.,$.$\rangle denotes the duality pairing between W^{-1 / t, t}(\Gamma)$ and $W^{1 / t, s}(\Gamma)$.

Now, we are able to formulate precisely the parabolic problem (1).

\subsection{Dual Mixed and Hybrid Finite Element Method DMHI}

In this section, we deal with the first variational formulation of the convection-diffusion problem previously considered. The principal objective of the following is to extend to the 2D case the first formulation proposed in (Fortin \& Serghini Mounim, 2005).

As in (Fortin \& Serghini Mounim, 2005), we shall begin from equation (1). Next, we introduce convective and diffusion fluxes as auxiliary variables to obtain the following first order system:

$$
\left\{\begin{array}{l}
\left.\frac{\partial u}{\partial t}-v \operatorname{div}(\underline{p})+\operatorname{div}(\underline{\hat{p}})=f \quad \text { in } \Omega \times\right] 0, T[ \\
\underline{p}=\operatorname{grad} u \quad \text { in } \Omega \times] 0, T[ \\
\underline{\hat{p}}=\underline{a} u \quad \text { in } \Omega \times] 0, T[
\end{array}\right.
$$

Boundary conditions and initial data are added to these equations. 
Here the boundary of $\Omega$ is supposed polygonal. Introducing $\lambda$, a Lagrange multiplier to enforce the continuity constraint of $\underline{q} . \underline{n}$ at elements' interfaces, we obtain

$$
\int_{\Omega} \underline{p} \cdot \underline{q} d x=-\sum_{K_{i}} \int_{K_{i}} u \operatorname{div}(\underline{q}) d x+\sum_{K_{i}} \int_{\partial K_{i}} \lambda \underline{q} \cdot \underline{n} d s .
$$

We shall evidently have to make a proper choice for the space of $\lambda$. This technique was first used by Fraejis DE Veubeke (Fraejis DE Veubeke, 1965; Fraejis DE Veubeke, 1975). In the same way, we shall impose the continuity of the convective flux $\hat{p}$ at the interfaces of elements $K_{i}$, with the aid of a Lagrange multiplier noted $\hat{\lambda}$. Hence, the last equation of the system (9) can now be rewritten as:

$$
\int_{\Omega} \underline{\hat{p}} \cdot \underline{q} d x=\sum_{K_{i}} \int_{K_{i}} \underline{a} \cdot \underline{q} d x+\sum_{K_{i}} \int_{\partial K_{i}} \hat{\lambda} \underline{q} \cdot \underline{n} d s .
$$

Now, to make the variational formulation precise we have to define the functional spaces. Let us consider an exponent $\frac{4}{3}<s<2$ and its conjugate $t$. In addition, we set $X=\left\{\underline{q} \in\left(L^{t}(\Omega)\right)^{2} ;\left.\underline{q}\right|_{K} \in W_{t}(\operatorname{div} ; K) \forall K \in T_{h}\right\}, M_{2}=\{\mu \in$ $\left.\prod_{K \in T_{h}} W^{1 / t, s}(\partial K) ;\left.\mu\right|_{\partial K \cap \Gamma}=0 \quad \forall K \in T_{h}\right\}$, and $M_{1}=L^{t}(\Omega)$. Hence, the system (9) could be written as follows:

Find $(u(t), p(t), \hat{p}(t)) \in M_{1} \times X^{2}$ and $(\lambda(t), \hat{\lambda}(t)) \in M_{2}^{2}$ such that

$$
\left\{\begin{array}{l}
\int_{\Omega} \frac{\partial u}{\partial t} v d x-v \sum_{K_{i}} \int_{K_{i}} \operatorname{div}(\underline{p}) v d x+ \\
\sum_{K_{i}} \int_{K_{i}} \operatorname{div}(\underline{\hat{p}}) v d x=\int_{\Omega} f v d x \quad \forall v \in M_{1}, \\
\int_{\Omega} \underline{p} \cdot \underline{q} d x=-\sum_{K_{i}} \int_{K_{i}} u \operatorname{div}(\underline{q}) d x+\sum_{K_{i}} \int_{\partial K_{i}} \lambda \underline{q} \cdot \underline{n} d s \quad \forall \underline{q} \in X, \\
\int_{\Omega} \underline{\hat{p}} \cdot \underline{q} d x=\sum_{K_{i}} \int_{K_{i}}(\underline{a} \cdot \underline{q} \underline{q}) d x+\sum_{K_{i}} \int_{K_{i}} \hat{\lambda} \underline{q} \cdot \underline{n} d s \quad \forall \underline{q} \in X, \\
\sum_{K_{i}} \int_{\partial K_{i}} \underline{p} \cdot \underline{n} \mu d s=0 \quad \forall \mu \in M_{2}, \\
\sum_{K_{i}} \int_{\partial K_{i}} \underline{\hat{p}} \cdot \underline{n} \mu d s=0 \quad \forall \mu \in M_{2} .
\end{array}\right.
$$

The last two equations of system (12) insure the continuity of $\underline{p} \cdot \underline{n}$ and $\underline{\hat{p}} \cdot \underline{n}$ at the interfaces of $T_{h}$.

\section{Remark 1}

The expressions $\int_{\partial K} \underline{q} \underline{n} \mu d s$ and $\int_{\partial K} \underline{\hat{q}} \underline{.} \underline{\mu} \mu d s$ are duality pairing between $W^{-1 / t, t}(\partial K)$ and $W^{1 / t, s}(\partial K)$. Indeed, $\left.\underline{q}\right|_{K} \in$ $\left(W_{t}(\operatorname{div} ; K)\right)^{2}$ and its normal trace is defined in $W^{-1 / t, t}(\partial K)$.

\section{Remark 2}

The proof of the existence of Lagrange multipliers $\lambda$ and $\hat{\lambda}$, which insure the continuity of normal traces is similar to the one given in Brezzi-Fortin (Brezzi \& Fortin, 1991).

Once the spaces are defined, we can establish the existence and uniqueness of problem (12) inspired from Girault-Raviart (Godlewski \& Raviart, 1991) (for details we can see (Serghini Mounim, 2000)).

Theorem 1 Let $u$ be the solution of the convection-diffusion problem (1). If $\underline{p}=\operatorname{grad} u$ and $\underline{\hat{p}}=\underline{\text { au verify }}$

$$
(\underline{p}(t), \underline{\hat{p}}(t)) \in\left(W_{t}(\operatorname{div} ; \Omega)\right)^{2},
$$

then $\left((\underline{p}, \underline{\hat{p}}, \hat{\lambda}) ;\left(u,\left.u\right|_{\partial K}\right)\right)$ is the unique solution of $(12)$. 


\subsection{Dual Mixed and Hybrid Finite Element DMH2}

Let us consider the following partial differential equation for $u$ :

$$
\left.\frac{\partial u}{\partial t}+\operatorname{div}(\underline{a} u)=0 \quad \text { in } \Omega \times\right] 0, T[,
$$

where $\underline{a}(x, t)$ is the velocity.

The dual mixed and hybrid formulation of the problem (14) can be achieved proceeding in a similar way as in (Fortin \& Serghini Mounim, 2005). First, we introduce again the convective flux as an auxiliary variable to obtain:

$$
\left\{\begin{array}{l}
\left.\frac{\partial u}{\partial t}+\operatorname{div}(\underline{\hat{p}})=0 \quad \text { in } \Omega \times\right] 0, T[ \\
\underline{\hat{p}}=\underline{a} u, \quad \text { in } \Omega \times] 0, T[.
\end{array}\right.
$$

Boundary conditions and initial data are added to these equations.

Next, we assume that the solution has discontinuities at each boundary of every $K_{i}$. Finally, we suggest that the "RankineHugoniot" jump condition should be imposed on the numerical flux through the elements' interfaces. With this aim, we employ a Lagrange multiplier to relax these jump conditions at the interelements.

$$
\int_{\Omega} \underline{\hat{p}} \cdot \underline{q} d x=\sum_{K_{i}} \int_{K_{i}} \underline{a} u \underline{q} \underline{q} d x+\sum_{K_{i}} \int_{\partial K_{i}} \hat{\lambda} \underline{q} \cdot \underline{n} d s \quad \forall \underline{q} \in X,
$$

and

$$
\sum_{K_{i}} \int_{\partial K_{i}}[\hat{p} . \underline{n}] \mu d s=\sum_{K_{i}} \int_{\partial K_{i}} \operatorname{sign}(\underline{a} . \underline{n}) a_{i}[u] \mu d s \quad \forall \mu \in M_{2} .
$$

We denote by $\hat{\lambda}$ the Lagrange multiplier and by $a_{i} \geq 0$ some approximation of the local propagation speeds. As we will see, it plays a stabilization parameter role, and for an appropriate choice of $a_{i}$, we can recover in particular the well-known upwind numerical fluxes (approximate Riemann solvers). Here, we have set $[\underline{\hat{p}} \cdot \underline{n}]=\hat{\hat{p}}^{i} \cdot \underline{n}-\underline{\hat{p}^{e}} \cdot \underline{n}$, where "the interior normal trace " $\underline{\hat{p}}^{i} \cdot \underline{n}$ (resp. "the exterior normal trace" $\underline{\hat{p}^{e}} \cdot \underline{n}$ ) is defined as the normal trace of the restriction $\left.\underline{\hat{p}}\right|_{K}$ (resp. $\left.\left.\hat{p}\right|_{(\Omega \backslash K)}\right)$ on $\partial K$, and with $[u]=u^{i}-u^{e}$ stands for the jump of $u$, where "the interior trace" $u^{i}$ and "the exterior trace" $u^{e}$ of $\bar{u}$ are defined from the trace of $u$ as above. In order to obtain the non-standard mixed hybrid finite element formulation, we keep the same notation for functional spaces as in the previous section. Thus, the variational formulation of (14) is to find $(u(t), \hat{\lambda}(t)) \in M_{1} \times M_{2}$ and $\hat{p}(t) \in X$ such that:

$$
\left\{\begin{array}{l}
\int_{\Omega} \frac{\partial u}{\partial t} v d x+\sum_{K_{i}} \int_{K_{i}} \operatorname{div}(\underline{\hat{p}}) v d x=0 \quad \forall v \in M_{1}, \\
\int_{\Omega} \underline{\hat{p}} \cdot \underline{q} d x=\sum_{K_{i}} \int_{K_{i}}(\underline{a} u \cdot \underline{q}) d x+\sum_{K_{i}} \int_{\partial K_{i}} \hat{\lambda} \underline{q} \cdot \underline{n} d s \quad \forall \underline{q} \in X, \\
\sum_{K_{i}} \int_{\partial K_{i}}[\hat{p} \cdot \underline{n}] \mu d s=\sum_{K_{i}} \int_{\partial K_{i}} \operatorname{sign}(\underline{a} \cdot \underline{n}) a_{i}[u] \mu d s \quad \forall \mu \in M_{2} .
\end{array}\right.
$$

The last equation of (18) imposes jump conditions to the flux $\hat{p} . \underline{n}$ at the interelement interfaces of $T_{h}$. We are now able to obtain a result of existence and uniqueness of the solution for problem (18), using an argument of regularity as in Theorem 2.23 .

Theorem 2 Consider $u$ the solution of the problem (14). If $\underline{\hat{p}}=\underline{\text { au }}$ satisfies

$$
\underline{\hat{p}}(t) \in W_{t}(\operatorname{div} ; \Omega),
$$

then $\left((\hat{\hat{p}}, \hat{\lambda}) ;\left(u,\left.u\right|_{\partial K}\right)\right)$ is the unique solution of $(18)$.

\section{Spatial Approximation}

Let us now deal with the finite element approximation of the above dual variational problem. To this aim, we assume that $\Omega$ is a polygonal bounded domain of R. Let $T_{h}$ be a standard finite element partition of $\Omega$. 


\subsection{Spatial Approximation of DMH1}

To define a discretization of problem (12), we first define the approximation spaces $X_{h}, M_{1 h}$ and $M_{2 h}$ of $X, M_{1}$ and $M_{2}$, respectively. We thus build an appropriate approximation space for $\underline{q} \in X$ in the standard way as follows:

$$
X_{h}=\left\{\underline{q}_{h} \in X ;\left.\quad \underline{q}_{h}\right|_{K} \in R T_{0}(K) \quad \forall K \in T_{h}\right\},
$$

where $R T_{0}(K)$ is the local Raviart-Thomas space of lowest degree.

To obtain the approximation of the spaces $M_{1}$ and $M_{2}$, we define

$$
M_{1 h}=\left\{v_{h} \in M_{1} ;\left.\quad v_{h}\right|_{K} \in \mathrm{P}_{0}(K) \quad \forall K \in T_{h}\right\},
$$

and

$$
M_{2 h}=\left\{\mu_{h} \in M_{2} ;\left.\quad \mu_{h}\right|_{\partial K} \in R_{0}(\partial K) \quad \forall K \in T_{h}\right\},
$$

where

$$
R_{0}(\partial K)=\left\{\varphi ;\left.\quad \varphi\right|_{e} \in \mathrm{P}_{0}(e) \quad \forall e \in \partial K\right\} .
$$

Finally, the discrete version of (12) is given by:

Find $\left(u_{h}(t), \lambda_{h}(t), \hat{\lambda}_{h}(t)\right) \in M_{1 h} \times M_{2 h}^{2}$ and $\left(p_{h}(t), \hat{p}_{h}(t)\right) \in X_{h}^{2}$ such that:

$$
\left\{\begin{array}{l}
\int_{\Omega} \frac{\partial u_{h}}{\partial t} v_{h} d x-v \sum_{K_{i}} \int_{K_{i}} \operatorname{div}\left(\underline{p}_{h}\right) v_{h} d x+ \\
\sum_{K_{i}} \int_{K_{i}} \operatorname{div}\left(\underline{\hat{p}}_{h}\right) v_{h} d x=\int_{\Omega} f v_{h} d x \quad \forall v_{h} \in M_{1 h}, \\
\int_{\Omega} \underline{p}_{h} \cdot \underline{q}_{h} d x=-\sum_{K_{i}} \int_{K_{i}} u_{h} \operatorname{div}\left(\underline{q}_{h}\right) d x+\sum_{K_{i}} \int_{\partial K_{i}} \lambda_{h} \underline{q}_{h} \cdot \underline{n} d s \quad \forall \underline{q}_{h} \in X_{h}, \\
\int_{\Omega} \underline{\hat{p}}_{h} \cdot \underline{q}_{h} d x=\sum_{K_{i}} \int_{K_{i}}\left(\underline{a}_{h} \cdot \underline{q}_{h}\right) d x+\sum_{K_{i}} \int_{\partial K_{i}} \hat{\lambda}_{h} \underline{q}_{h} \cdot \underline{n} d s \quad \forall \underline{q}_{h} \in X_{h}, \\
\sum_{K_{i}} \int_{\partial K_{i}} \underline{p}_{h} \cdot \underline{n} \mu_{h} d s=0 \quad \forall \mu_{h} \in M_{2 h}, \\
\sum_{K_{i}} \int_{\partial K_{i}} \hat{p}_{h} \cdot \underline{n} \mu_{h} d s=0 \quad \forall \mu_{h} \in M_{2 h} .
\end{array}\right.
$$

To these equations we have to add given initial data, and corresponding suitable boundary conditions.

\section{Theorem 3 Problem (23) has a unique solution.}

Proof. Solving the problem (23) leads to solve a square finite-dimensional system of linear algebraic equations. Hence, we only have to prove the uniqueness of the solution.

If we consider the homogeneous problem associated to (23), $\mathrm{f}=0$, and if the boundary conditions and initial value vanish, the proof is similar to that of the Theorem 1, in regard to $u_{h}, \underline{p}_{h}$ and $\underline{\hat{p}}_{h}$. For the uniqueness of $\lambda_{h}$ and $\hat{\lambda}_{h}$, we consider

$$
\sum_{K_{i}} \int_{\partial K_{i}} \underline{q}_{h} \cdot \underline{n} \lambda_{h} d s=0 \quad \forall \underline{q}_{h} \in M_{1 h},
$$

let

$$
\int_{\partial K_{i}} \underline{q}_{h} \cdot \underline{n} \lambda_{h} d s=0 \quad \forall \underline{q}_{h} \in R T_{0}\left(K_{i}\right)
$$

and we then deduce that $\lambda_{h}=0$, and similarly we have $\hat{\lambda}_{h}=0$. 


\subsection{Spatial Approximation of DMH2}

To define the associated discrete problem of (18), we may now use the same suitable subspaces as above, and we also proceed in the same manner. Hence, we obtain the following discrete problem:

Find $\left(u_{h}(t), \hat{\lambda}_{h}(t)\right) \in M_{1 h} \times M_{2 h}$ and $\hat{p}_{h}(t) \in X_{h}$ such that

$$
\left\{\begin{array}{l}
\int_{\Omega} \frac{\partial u_{h}}{\partial t} v_{h} d x+\sum_{K_{i}} \int_{K_{i}} \operatorname{div}\left(\underline{\hat{p}}_{h}\right) v_{h} d x=0 \quad \forall v_{h} \in M_{1 h}, \\
\int_{\Omega} \underline{\hat{p}}_{h} \cdot \underline{q}_{h} d x=\sum_{K_{i}} \int_{K_{i}}\left(\underline{a} u_{h} \cdot \underline{q}_{h}\right) d x+\sum_{K_{i}} \int_{\partial K_{i}} \hat{\lambda}_{h} \underline{q}_{h} \cdot \underline{n} d s \quad \forall \underline{q}_{h} \in X_{h}, \\
\sum_{K_{i}} \int_{\partial K_{i}}\left[\underline{\hat{p}}_{h} \cdot \underline{n}\right] \mu_{h} d s=\sum_{K_{i}} \int_{\partial K_{i}} \operatorname{sign}(\underline{a} \cdot \underline{n}) a_{i}\left[u_{h}\right] \mu_{h} d s \quad \forall \mu_{h} \in M_{2 h},
\end{array}\right.
$$

$a_{i}$ is again the approximation of the local velocity at $\partial K_{i},\left(a_{i} \geq 0\right)$. In a similar way as we did for Theorem 3 , we can prove the following result:

Theorem 4 The problem (24) has a unique solution.

\section{Analogy with the Finite Volume Method}

The contents of this section are discussed in more details in (Serghini Mounim, 2000). Notice that the most attractive property of finite volume methods is that the conservation of quantities such as mass, momentum and energy is exactly satisfied on each finite volume and also on the whole domain. To make the link explicit with finite volume methods, we use the $R T$ vector finite elements to approximate the diffusion and convective fluxes. We point out this relationship, using a suitable quadrature formula to approximate the terms $\int_{K} \underline{p} \cdot \underline{q} d x$ and $\int_{K} \underline{f}(u) \cdot \underline{q} d x, \underline{f}(u)$ denotes here the convective flux.

\subsection{DMHI and scheme1}

\subsubsection{Rectangular Case}

We consider a quadrangulation $T_{h}$ of $\Omega$, and we denote by $K$ the generic rectangle of $T_{h}$ defined by $\left[x^{K}, x^{K}+\Delta x^{K}\right] \times$ $\left[y^{K}, y^{K}+\Delta y^{K}\right]$, and the degrees of freedom introduced by Raviart-Thomas. The usual $R T$ basis functions of $K$ are defined by:

$$
\left\{\begin{array}{l}
p_{S}=\frac{1}{|K|}\left(0, y-y^{K}-\Delta y^{K}\right), \\
p_{E}=\frac{1}{|K|}\left(x-x^{K}, 0\right), \\
p_{N}=\frac{1}{|K|}\left(0, y-y^{K}\right), \\
p_{W}=\frac{1}{|K|}\left(x-x^{K}-\Delta x^{K}, 0\right),
\end{array}\right.
$$

where $|K|$ is the area of $K$.

In order to diagonalize the mass matrix $\int_{K} \underline{p} \cdot \underline{q} d x$, still retaining the good numerical properties of the mixed approximation, we employ the trapezoidal quadrature rule to obtain the following diagonal matrix:

$$
\frac{1}{2}\left(\begin{array}{cccc}
\frac{\Delta y}{\Delta x} & 0 & 0 & 0 \\
0 & \frac{\Delta x}{\Delta y} & 0 & 0 \\
0 & 0 & \frac{\Delta y}{\Delta x} & 0 \\
0 & 0 & 0 & \frac{\Delta x}{\Delta y}
\end{array}\right) .
$$

For simplicity, we suppose $f=0$, and in what follows we shall write $u, p, \hat{p}$ instead $u_{h}, p_{h}, \hat{p}_{h}, \ldots$ and omit the dependance of index sets $u, p, \hat{p}, \ldots$ on $\mathrm{h}$. Now, let us fix our attention on two adjacent rectangles $K$ and $K_{i}, i \in\{S, E, N, W\}$, and denote the values of $u$ in $K$ and $K_{i}$ by $u_{K}$ and $u_{i}$ respectively. Next, as in the 1D case (see (Fortin \& Serghini Mounim, 2005)) a direct computation provides:

$$
\lambda_{i}=\frac{u_{K}+u_{i}}{2},
$$


and

$$
\hat{\lambda}_{i}=\frac{|K|}{4} \frac{f\left(u_{i}\right)-\underline{f}\left(u_{K}\right)}{\left|e_{i}\right|} \cdot \underline{n}_{i},
$$

from which we can deduce the approximation of the convective flux at the faces of $K$

$$
\hat{\alpha}_{i}=\frac{\left|e_{i}\right|}{2}\left(\underline{f}\left(u_{K}\right)+\underline{f}\left(u_{i}\right)\right) \cdot \underline{n}_{i},
$$

where the outward normal to the faces $e_{i}$ of $K$ is denoted by $\underline{n}_{i}$, with $\left|e_{i}\right|$ stands for the length of $e_{i}$ for $i \in\{S, E, N, W\}$.

Then, we observe that the expressions of $\hat{\alpha}_{i}$ are equivalent to a finite volume method. Indeed, we obtain a "central difference scheme" (CDS). This scheme is not spatially stable; it does not prevent oscillations near discontinuities and at shocks, (for the properties of CDS see Lazarov et al (Lazarov, \& Vassilevski, 1996)).

\subsubsection{Other Formulation and Upwind Schemes}

If we impose the continuity of convective flux only at inflow boundary of each control volume $K$, we can obtain an upwind scheme. For this purpose, we utilize the following form:

$$
\begin{aligned}
c\left(\left(\hat{p}_{h}, \hat{\lambda}\right) ; q_{h}\right) & =\int_{\Omega} \hat{p}_{h} \cdot q_{h} d x-\sum_{K_{i}} \int_{K_{i}} f\left(u_{h}\right) \cdot q_{h} d x+\sum_{K_{i}} \int_{\partial K_{-}} \hat{\lambda} \underline{q} \cdot \underline{n} d s, \\
& =0 \quad \forall q_{h} \in X_{h} .
\end{aligned}
$$

$\partial K_{-}$denotes the inflow boundary of $K$, which is defined by

$$
\partial K_{-}=\{x \in \partial K \quad: \quad \underline{a} \cdot \underline{n} \leq 0\} .
$$

Using the same techniques as in the previous subsection, we can derive easily the numerical convective flux at the interface between adjacent cells:

$$
\hat{\alpha}_{i}=\left|e_{i}\right|\left[\left(\frac{\underline{f}\left(u_{K}\right)+\underline{f}\left(u_{i}\right)}{2}\right) \cdot \underline{n}_{i}-\frac{1}{2}\left|\left(\frac{\partial \underline{f}}{\partial u}\right)_{\underline{u}} \cdot \underline{n}_{i}\right|\left(u_{i}-u_{K}\right)\right],
$$

since

$$
\underline{f}\left(u_{i}\right)-\underline{f}\left(u_{K}\right)=\left(\frac{\partial \underline{f}}{\partial u}\right)_{\underline{u}}\left(u_{i}-u_{K}\right),
$$

where $\underline{u}$ represents some average state between $u_{i}$ and $u_{K}$.

For the linear convection case $(\underline{f}(u)=\underline{a} u$ where $\underline{a}$ denotes again the convective field), the above expression then reads:

$$
\hat{\alpha}_{i}=\left|e_{i}\right|\left[\frac{\underline{f}\left(u_{K}\right) \cdot \underline{n}_{i}+\left|\underline{f}\left(u_{K}\right) \cdot \underline{n}_{i}\right|}{2}+\frac{\underline{f}\left(u_{i}\right) \cdot \underline{n}_{i}-\left|\underline{f}\left(u_{i}\right) \cdot \underline{n}_{i}\right|}{2}\right],
$$

where $i \in\{S, E, N, W\}$, and $\left|e_{S}\right|=\left|e_{N}\right|=\Delta x,\left|e_{E}\right|=\left|e_{W}\right|=\Delta y$. This scheme coincides with the standard UDS (upwind difference scheme) see for example (LeVeque, 2002).

Before considering the generalization of the previous schemes, we note that one advantage of the use of $\hat{\lambda}$ is that no (approximate) Riemann solvers are required.

\section{Remark 3}

Since $u$ is discretized using piecewise polynomial functions and our formulation involves the boundary integrals, we can introduce an upwinding in the formulation. To achieve the generalization of the schemes above, we propose the following form

$$
\sum_{K}\left\{\frac{1+\beta}{2} \int_{\partial K_{-}} \hat{\lambda} \underline{q} \cdot \underline{n} d s+\frac{1-\beta}{2} \int_{\partial K_{+}} \hat{\lambda} \underline{q} \cdot \underline{n} d s\right\}
$$

to replace the form already used $\sum_{K} \int_{\partial K} \hat{\lambda} \underline{q} \cdot \underline{n} d s$ in the system (23). The following notation is used:

$$
\partial K_{+}=\{x \in \partial K \quad: \quad \underline{a} \cdot \underline{n}>0\} .
$$


Taking the same approach as above, this results in the generalized upwind scheme:

$$
\begin{aligned}
\sum_{i=1}^{4} \hat{\alpha}_{i}= & \Delta x\left((1-\beta) \frac{f_{2}\left(u_{N}\right)-f_{2}\left(u_{S}\right)}{2}+2 \beta \frac{f_{2}\left(u_{K}\right)-f_{2}\left(u_{S}\right)}{2}\right)+ \\
& \Delta y\left((1-\beta) \frac{f_{1}\left(u_{E}\right)-f_{1}\left(u_{W}\right)}{2}+2 \beta \frac{f_{1}\left(u_{K}\right)-f_{1}\left(u_{W}\right)}{2}\right) .
\end{aligned}
$$

Here $f=\left(f_{1}, f_{2}\right)$, and $\beta$ is the dissipation parameter $(0 \leq \beta \leq 1)$ which controls the combination of fully upwind and centered scheme. We have supposed without loss of generality that the inflow boundary of $K$ is $e_{S} \cup e_{W}$. Notice that for $\beta=1$, we recover the scheme (29), (30).

\subsubsection{Triangular Case}

We consider now the case where $T_{h}$ is a standard triangulation of $\Omega$ (here, we suppose again that $\Omega$ is a polygonal bounded domain of R). We begin with a brief description of the notation used here. For each triangle $K$, we have:

- $|K|$ : area of $K$;

- $\underline{a}_{r}: r^{\text {th }}$ vertex of $K$ with coordinates $\left(x_{r}, y_{r}\right)$;

- $e_{r}$ : face opposite to vertex $a_{r}$, with length $\left|e_{r}\right|$;

- $h_{r}$ : the length of the perpendicular dropped from the vertex $\underline{a}_{r}$;

- $\underline{n}_{r}$ : unit exterior normal to face $e_{r}$;

- $\theta_{r}$ : angle at vertex $a_{r}$;

for $r \in\{i, j, k\}$.

We also consider the local shape functions $\left\{p_{r}\right\}_{r \in\{i, j, k\}}$ of $R T_{0}(K)$ for every triangle $K$. The degrees of freedom are the fluxes through the faces $\left\{e_{r}\right\}_{r \in\{i, j, k\}}$, and are defined by:

$$
\underline{p}_{r}(\underline{x})=\frac{\underline{x}-\underline{a}_{r}}{2|K|} \equiv \frac{\underline{x}-\underline{a}_{r}}{h_{r}\left|e_{r}\right|} \quad \forall \underline{x}=\left(x_{1}, x_{2}\right) \in K
$$

from which, for every local vectorial function $\underline{p}_{r}$ of $R T_{0}(K)$ the well known properties follow:

$$
\left\{\begin{array}{l}
\operatorname{supp}\left(\underline{p}_{r}\right)=K \cup K^{\prime}, \quad \text { with } K \cap K^{\prime}=e_{r}, \\
\operatorname{div}\left(\underline{p}_{r}\right)=\frac{1}{|K|}, \\
\left.\underline{p}_{r} \cdot \underline{n}_{s}\right|_{e_{s}}=\frac{\delta_{r s}}{\left|e_{r}\right|},
\end{array}\right.
$$

where $\delta_{r s}$ denotes the Kronecker symbol $\delta_{r s}= \begin{cases}1 & \text { if } r=s, \\ 0 & \text { otherwise. }\end{cases}$

We introduce the bilinear form $a(\underline{p}, \underline{q}) \equiv \int_{K} \underline{p} \underline{q} d x$ for all $\underline{p}, \underline{q}$ in $R T_{0}(K)$. Then, $a\left(\underline{p}_{r}, \underline{p}_{s}\right)$ is a $3 \times 3$ symmetric, positive definite matrix,

$$
a\left(\underline{p}_{r}, \underline{p}_{s}\right) \equiv a_{r s}=\frac{1}{12}\left(\begin{array}{ccc}
3 c_{i}+c_{j}+c_{k} & c_{k}-\left(c_{i}+c_{j}\right) & c_{j}-\left(c_{k}+c_{i}\right) \\
c_{k}-\left(c_{i}+c_{j}\right) & 3 c_{j}+c_{k}+c_{i} & c_{i}-\left(c_{j}+c_{k}\right) \\
c_{j}-\left(c_{k}+c_{i}\right) & c_{i}-\left(c_{j}+c_{k}\right) & 3 c_{k}+c_{i}+c_{j}
\end{array}\right)
$$

with $c_{r} \equiv \cot \theta_{r}, \quad$ for $r, s \in\{i, j, k\}$. 


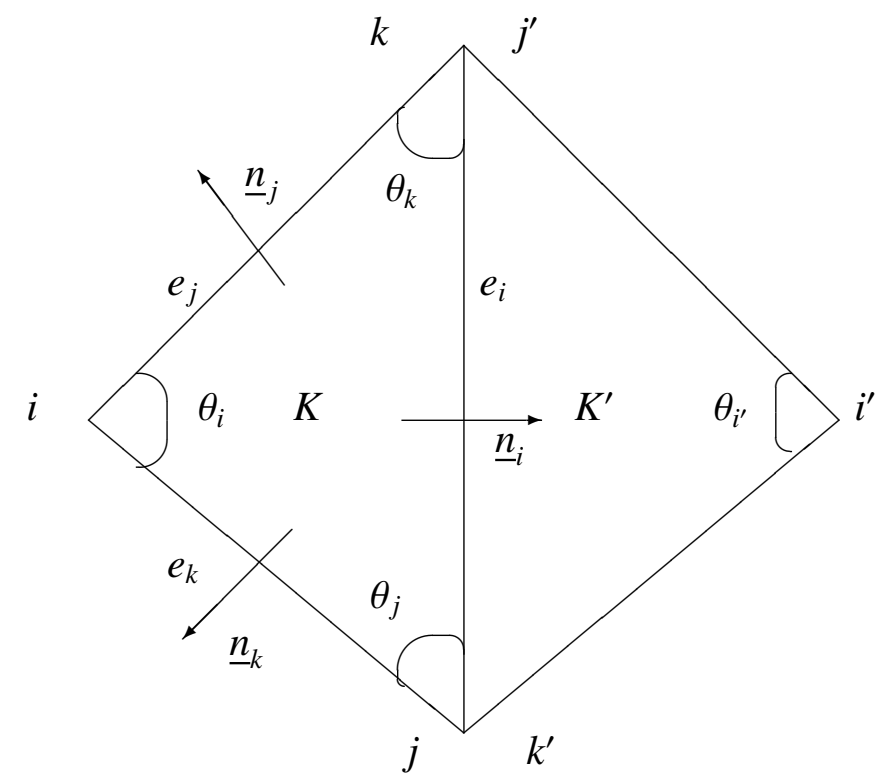

Figure 1. Notations for triangles $K$ and $K^{\prime}$

We employ the quadrature formula proposed in (Barranger, Maitre, \& Oudin, 1996), to diagonalize the local mass matrix (34). We obtain the matrix:

$$
a_{r s}^{h} \equiv \frac{1}{2} \delta_{r s} \cot \theta_{r}, \quad \text { for } r, s \in\{i, j, k\} .
$$

From this, after temporal approximation, and considering the neighbor elements of $K$ which appear in the formula, the equation expressing the discrete conservation law becomes:

$$
|K| \frac{u_{K}^{n+1}-u_{K}^{n}}{\delta t}-v \sum_{r=i, j, k} \alpha_{r}+\sum_{r=i, j, k} \hat{\alpha}_{r}=0,
$$

and thanks to the following relation,

$$
\int_{K} \underline{p} \cdot \underline{q} d x=-\int_{K} u \operatorname{div}(\underline{q}) d x+\int_{\partial K} \lambda \underline{q} \cdot \underline{n} d s,
$$

we obtain

$$
\beta_{r} \alpha_{r}=-u_{K}+\lambda_{r}
$$

where we have set $\beta_{r}=\frac{1}{2} \cot \theta_{r}, \quad$ with $r=i, j, k$.

From the above equation, in conjunction with the continuity of the normal trace of the diffusive flux, we deduce

$$
\lambda_{r}=\frac{\beta_{r^{\prime}}}{\beta_{r}+\beta_{r^{\prime}}} u_{K}+\frac{\beta_{r}}{\beta_{r}+\beta_{r^{\prime}}} u_{K_{r}}, \quad r=i, j, k .
$$

Using (37) and (38), the numerical diffusion flux across edge $e_{r}$ reads

$$
\alpha_{r}=\frac{u_{K_{r}}-u_{K}}{\beta_{r}+\beta_{r^{\prime}}}
$$

We note that $\beta_{r}=\frac{d_{r}}{\left|e_{r}\right|}, r=i, j, k$ (see the annexe in (Bank \& Rose, 1987; Kerkhoven-Jerome, 1990)), where $d_{r}$ denotes the distance between the circumscribed circle of $K$ and the center $m_{r}$ on sides $e_{r}$ (see previous and below figures). We mention that $\beta_{r}$ is also given by $\beta_{r}=-\frac{\left|e_{s}\right| t_{s} \cdot\left|e_{s^{\prime}}\right| \underline{t}_{s^{\prime}}}{4|K|}$, where we have set $s=I(r), s^{\prime}=I(s)$, with $I$ defined as $I(i)=j, I(j)=k$ and $I(k)=i$, and $\underline{t}_{r}$ stands for the unit tangent to $e_{r}$ for $r=i, j, k$. 


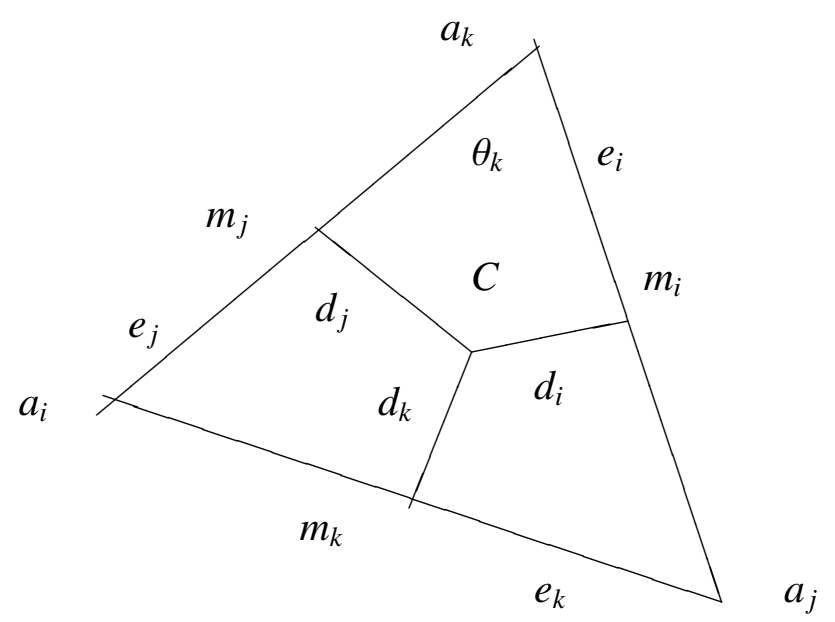

Figure 2. Circumscribed circle and distances $d_{i}$.

With the aid of the new expression of $\beta_{r}$ the system (39) becomes

$$
\alpha_{r}=\left|e_{r}\right| \frac{u_{K_{r}}-u_{K}}{d_{r}+d_{r^{\prime}}}, \quad r=i, j, k,
$$

where $d_{r}+d_{r^{\prime}}$ is the distance between the circumcenters $C, C_{r}$ of two adjoining triangles $K$ and $K_{r}$. The above distance does not vanish, in the case of a strictly acute triangle.

We now deal with the convective flux using the same notation and procedure as above. We thus start from the equation

$$
\int_{K} \underline{\hat{p}} \cdot \underline{q} d x=\int_{K} \underline{f}(u) \cdot \underline{q} d x+\int_{\partial K} \hat{\lambda} \underline{q} \cdot \underline{n} d s .
$$

We approximate the mass matrix with the same quadrature formula as in the diffusive case, in addition, we use the following numerical integration which is exact for polynomials of degree 0 :

$$
\int_{K} p(x, y) d x d y \simeq|K| p\left(H_{K}\right)
$$

where $H_{K}$ is the orthocenter of the triangle $K$ which is considered acute, and $|K|$ denotes the area of $K$ as illustrated in Figure 3.

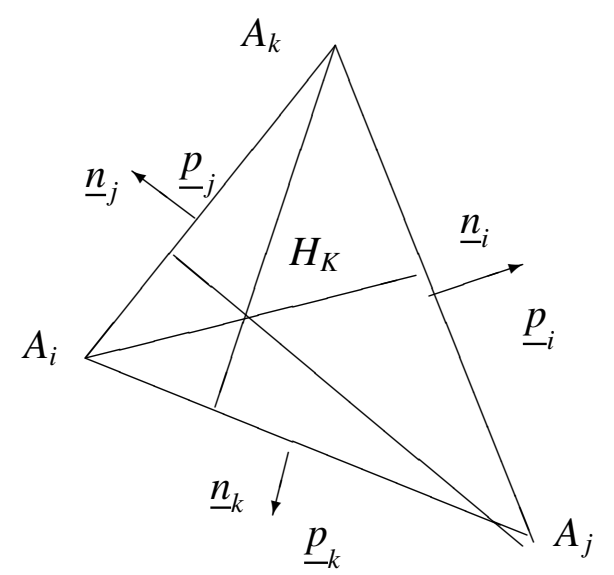

Figure 3. The orthocenter $H_{K}$ of the triangle $K$.

Thanks to the above formula (41), the integral $\int_{K} \underline{f}_{(u)} \underline{p}_{r} d x$ is approximated by

$$
\int_{K} \underline{f}(u) \cdot \underline{p}_{r} d x \simeq \frac{f(u)}{2} \cdot \overrightarrow{A_{r} H_{K}}, \quad r=i, j, k .
$$


Finally, these approximate relations allow us to write the numerical convective flux as

$$
\hat{\alpha}_{r}=\frac{\left|e_{r}\right|}{2} \frac{f\left(u_{K}\right)\left\|\overrightarrow{A_{r} H_{K}}\right\|+\underline{f}\left(u_{K_{r}}\right)\left\|\overrightarrow{A_{r^{\prime}} H_{K_{r}}}\right\|}{d_{r}+d_{r^{\prime}}} . \underline{n}_{r}, \quad r=i, j, k,
$$

since $\beta_{r}=\frac{d_{r}}{\left|e_{r}\right|}$, for all $r \in\{i, j, k\}$.

If we only use equilateral triangles, we get $\left\|\overrightarrow{A_{r} H_{K}}\right\|=\left\|\overrightarrow{A_{r^{\prime}} H_{K_{r}}}\right\|=2 d$, with $d_{r}=d_{r^{\prime}}=d$, and the approximation formula (42), becomes

$$
\hat{\alpha}_{r}=\left|e_{r}\right|\left(\frac{f\left(u_{K}\right)+\underline{f}\left(u_{K_{r}}\right)}{2}\right) \cdot \underline{n}_{r}, \quad r=i, j, k .
$$

It is precisely the central finite volume scheme "CDS".

\section{Remark 4}

In view of the linear case $\underline{f}(u)=\underline{a} u$, we can define $\underline{a}$ as a linear combination of the local shape functions $\underline{p}_{r}$ of $R T_{0}(K)$, after some manipulation, we can exhibit the numerical flux through the face $e_{r}$

$$
\hat{\alpha}_{r}=\frac{\left|e_{r}\right|}{d_{r}+d_{r^{\prime}}} \frac{u_{K}+u_{K_{r}}}{2} a_{h_{r}}, \quad r=i, j, k,
$$

where $\underline{a}_{h}=\sum_{r=i}^{k} a_{h_{r}} \underline{p}_{r}$. For other approximations the reader can refer to (Serghini Mounim, 2000).

\section{Remark 5}

If we utilize the bilinear form (31) and the quadrature formula (41), the numerical convective flux (43) becomes

$$
\hat{\alpha}_{r}=\left|e_{r}\right|\left[\frac{(1+\beta) \underline{f}\left(u_{K}\right)+(1-\beta) \underline{f}\left(u_{K_{r}}\right)}{2}+\beta \Xi\left(\underline{f}\left(u_{K}\right)-\underline{f}\left(u_{K_{r}}\right)\right)\right] \cdot \underline{n}_{r},
$$

for $r \in\{i, j, k\}$, with $\Xi=\min \left(\operatorname{sign}\left(\underline{a} \underline{n}_{r}\right), 0\right)$ and $\beta$ being again the upwinding parameter $(0 \leq \beta \leq 1)$. Note that for $\beta=1$ (or the continuity constraint imposed to $\hat{p}$ only at the inflow boundary of $K$ ), we get the following upwind finite volume scheme:

$$
\hat{\alpha}_{r}=\left|e_{r}\right|\left[\underline{f}\left(u_{K}\right)+\Xi\left(\underline{f}\left(u_{K}\right)-\underline{f}\left(u_{K_{r}}\right)\right)\right] \cdot \underline{n}, \quad r=i, j, k,
$$

for the stability and error estimates of CDS and UDS on Voronoi meshes we refer to (Mishev, 1998).

\subsection{DMH2 and Scheme2}

The results of this section are derived in a similar fashion to DMH1 case.

\subsubsection{Rectangular Case}

Consider again the case of a rectangular mesh $T_{h}$ of $\Omega$, and the local degrees of freedom associated to $R T_{0}(K)$. Here we denote by $\underline{n}_{r}^{e}$ (resp. $\underline{n}_{r}^{i}$ ) the exterior unit normal vector, i.e., oriented from $K$ to $K_{r}$ (resp. interior unit normal vector, i.e., oriented from $K_{r}$ to $K$ ) along each edge $e_{r}=\partial K \cap \partial K_{r}$; clearly, the property $\underline{n}_{r}^{e}=-\underline{n}_{r}^{i}$ holds. Now, following the same lines as in previous sections, we can state the result:

Proposition 2 If we use the trapezoidal quadrature formula to diagonalize the element mass matrix, and exact computation of the spatial integral $\int_{K} \underline{f}(u) \cdot \underline{q} d x$; then, the exterior $\hat{\alpha}_{r}^{e}$ and the interior $\hat{\alpha}_{r}^{i}$ fluxes approximating $\int_{e_{r}} \hat{\hat{p}}^{e} \cdot \underline{n}_{r}^{e} d s$ and $\int_{e_{r}} \underline{\hat{p}}^{i} \cdot \underline{n}_{r}^{e} d s$ respectively across edge $e_{r}$ are given by:

$$
\hat{\alpha}_{r}^{e}=\frac{\left|e_{r}\right|}{2}\left(\left(\underline{f}\left(u_{K}\right)+\underline{f}\left(u_{r}\right)\right) \cdot \underline{n}_{r}^{e}+\left.\operatorname{sign}\left(\underline{a} \cdot \underline{n}_{r}^{e}\right) a_{r}\right|_{e_{r}}\left(u_{r}-u_{K}\right)\right),
$$

and

$$
\hat{\alpha}_{r}^{i}=\frac{\left|e_{r}\right|}{2}\left(\left(\underline{f}\left(u_{K}\right)+\underline{f}\left(u_{r}\right)\right) \cdot \underline{n}_{r}^{e}+\left.\operatorname{sign}\left(\underline{a} \cdot \underline{n}_{r}^{e}\right) a_{r}\right|_{e_{r}}\left(u_{K}-u_{r}\right)\right),
$$

where $\hat{\lambda}_{r}$ is given by:

$$
\hat{\lambda}_{r}=\frac{|K|}{4} \frac{\left(\underline{f}\left(u_{r}\right)-\underline{f}\left(u_{K}\right)\right) \cdot \underline{n}_{r}^{e}+\left.\operatorname{sign}\left(\underline{a} \cdot \underline{n}_{r}^{e}\right) a_{r}\right|_{e_{r}}\left(u_{K}-u_{r}\right)}{\left|e_{r}\right|}
$$


for $r \in\{S, E, N, W\}$, and where $\left.a_{r}\right|_{e_{r}}$ stands for an approximation of the propagation speed at the edge $e_{r}$.

Hence, we can state that the Rankine-Hugoniot jump condition at $e_{r}$ is recovered in the expression of Lagrange multipliers. It is also important to notice that assuming that $e_{r}$ is the upstream boundary of $\partial K$, i.e., $\left.\operatorname{sign}\left(\underline{a} . \underline{n}_{r}^{e}\right)\right|_{e_{r}}<0$ (resp. $e_{r}$ is the downstream boundary of $\partial K$ ), then the numerical fluxes used in the FV methods at $e_{r}$ are our exterior fluxes: $F_{i}=\alpha_{r}^{e}$ (resp. our interior fluxes $\hat{\alpha}_{r}^{i}$ ). Then we can generalize the above formulation taking the same approach used in (Fortin $\&$ Serghini Mounim, 2005). To this end, we consider the values of the trace normal of the fluxes $\hat{p} . \underline{n}$ at the boundary of element $\partial K$ as a convex combination of the exterior fluxes $\underline{\hat{p}^{e}} . \underline{n}$ and the interior fluxes $\underline{\hat{p}^{i}} . \underline{n}$ :

$$
\underline{\hat{p}} \cdot \underline{n}= \begin{cases}\frac{1+\beta}{2} \hat{\hat{p}}^{e} \cdot \underline{n}+\frac{1-\beta}{2} \hat{\hat{p}}^{i} \cdot \underline{n} & \text { on } \partial K^{-} \\ \frac{1-\beta}{2} \underline{\hat{p}}^{e} \cdot \underline{n}+\frac{1+\beta}{2} \underline{\hat{p}}^{i} \cdot \underline{n} & \text { on } \partial K^{+}\end{cases}
$$

where $\beta \in[0,1]$ is the upwinding parameter, and $\underline{n}$ is again the exterior unit normal vector oriented from $K$ to neighboring triangles $K_{r}$. Consequently, by means of (44), we also obtain the local conservativity of the scheme (the numerical fluxes are obviously consistent). Hence, we replace in each $K_{i}, \int_{K_{i}} \operatorname{div}(\underline{p}) v_{h} d x$ by

$$
\left.\int_{\partial K_{i}^{-}}\left(\frac{1+\beta}{2} \underline{\hat{p}}^{e} \cdot \underline{n}+\frac{1-\beta}{2} \underline{\hat{p}}^{i} \cdot \underline{n}\right) v_{h} d s+\int_{\partial K_{i}^{+}}\left(\frac{1+\beta}{2} \underline{\hat{p}}^{i} \cdot \underline{n}\right)+\frac{1-\beta}{2} \underline{\hat{p}}^{e} \cdot \underline{n}\right) v_{h} d s .
$$

We mention that the usual expressions of the finite volume numerical fluxes (see for example (LeVeque, 2002; Beux et al, 1993) are obtained via our formulation, taking $\beta=1$ and for the choice $\beta=0$ we recover the centered scheme. Now, it is clear that the appropriate choice of the speed $a_{i}$ allows us to recover the standard volume schemes. Next, we focus our attention to giving the extensions of some recent schemes.

\section{Remark 6}

In the following we shall use in each $K$ the piecewise polynomial interpolant at time $t^{n}, P^{K}\left(., t^{n}\right)$, and the local velocities of propagation $a_{r}^{n}$, proposed in (Kurganov \& Levy, 2000). Next, taking a dimension by dimension approach, we can recover the two-dimensional, third order, non oscillatory scheme of Kurganov-Levy scheme, computing the interior and exterior traces of $u, u_{r}^{i, e}$ at the faces $e_{r}=\partial K \cap \partial K_{r}, r \in\{S, E, N, W\}$ following the recipe. The recipe is as follows (the computation of the exterior values $u_{r}^{e}$ can be carried out in the similar way, using $P^{r}\left(., t^{n}\right)$ in the neighboring rectangles)

$$
u_{r}^{i}=w_{L} P_{L}^{K}\left(x_{r} ; t^{n}\right)+w_{R} P_{R}^{K}\left(x_{r} ; t^{n}\right)+w_{C} P_{C}^{K}\left(x_{r} ; t^{n}\right), r \in\{W, E\},
$$

and

$$
u_{r}^{i}=w_{L} P_{L}^{K}\left(y_{r} ; t^{n}\right)+w_{R} P_{R}^{K}\left(y_{r} ; t^{n}\right)+w_{C} P_{C}^{K}\left(y_{r} ; t^{n}\right), r \in\{S, N\},
$$

where the polynomials $P_{L}^{K}, P_{R}^{K}, P_{C}^{K}$, and the weights $w_{L}, w_{R}, w_{C}$ are also introduced in (Kurganov \& Levy, 2000). Now, if we utilize $u_{r}^{i}$ and $u_{r}^{e}$ in (24), and thanks to Proposition 4.21, and the help to (44), this will result in the 2D extension Kurganov-Levy semi-discrete scheme.

\subsubsection{Triangular Case}

We consider now the case where $T_{h}$ is a triangular mesh. For each triangle $K$, we use the same notations as DMH1 case and we summarize the result obtained as follows:

Proposition 3 If we use the quadrature formula proposed in (Baranger, Maitre, $\mathcal{F}$ Oudin, 1996) to diagonalize the element mass matrix and we employ the numerical integration formula (41) then, the fluxes $\hat{\alpha}_{r}^{e}$ and $\alpha_{r}^{i}$ through the face $e_{r}$ are approximated by

$$
\begin{aligned}
\hat{\alpha}_{r}^{e} & =\frac{\left|e_{r}\right|}{d_{r}+d_{r^{\prime}}}\left(\frac{\left\|\overrightarrow{A_{r} H_{K}}\right\| \underline{f}\left(u_{K}\right) \cdot \underline{n}_{r}^{e}+\left\|\overrightarrow{A_{r^{\prime}} H_{K_{r}}}\right\| \underline{f}\left(u_{K_{r}}\right) \cdot \underline{n}_{r}^{e}}{2}\right. \\
& \left.+\left.\operatorname{sign}\left(\underline{a} \cdot \underline{n}_{r}^{e}\right) d_{r} a_{r}\right|_{e_{r}}\left(u_{K_{r}}-u_{K}\right)\right), \\
\hat{\alpha}_{r}^{i} & =\frac{\left|e_{r}\right|}{d_{r}+d_{r^{\prime}}}\left(\frac{\left\|\overrightarrow{A_{r} H_{K}}\right\| \underline{f}\left(u_{K}\right) \cdot \underline{n}_{r}^{e}+\left\|\overrightarrow{A_{r^{\prime}} H_{K_{r}}}\right\| \underline{f}\left(u_{K_{r}}\right) \cdot \underline{n}_{r}^{e}}{2}\right. \\
& \left.+\left.\operatorname{sign}\left(\underline{a} \cdot \underline{n}_{r}^{e}\right) d_{r^{\prime}} a_{r}\right|_{e_{r}}\left(u_{K}-u_{K_{r}}\right)\right),
\end{aligned}
$$


where $\hat{\lambda}_{r}$ is given by:

$$
\begin{aligned}
\hat{\lambda}_{r} & =-\frac{d_{r^{\prime}}}{d_{r}+d_{r^{\prime}}}\left\|\overrightarrow{A_{r} H_{K}}\right\| \frac{f\left(u_{K}\right)}{2} \cdot \underline{n}_{r}^{e}+\frac{d_{r}}{d_{r}+d_{r^{\prime}}}\left\|\overrightarrow{A_{r} H_{K_{r}}}\right\| \frac{f\left(u_{K_{r}}\right)}{2} \cdot \underline{n}_{r}^{e}, \\
& +\left.\operatorname{sign}\left(\underline{a} \cdot \underline{n}_{r}^{e}\right) \frac{d_{r} d_{r^{\prime}}}{d_{r}+d_{r^{\prime}}} a_{r}\right|_{r_{r}}\left(u_{K}-u_{K_{r}}\right),
\end{aligned}
$$

for $r=i, j, k$.

If we restrain to equilateral triangles, one then gets obviously $\left\|\overrightarrow{A_{r} H_{K}}\right\|=\left\|\overrightarrow{A_{r^{\prime}} H_{K_{r}}}\right\|=2 d$, with $d_{r}=d_{r^{\prime}}=d$, and, in particular, the approximation of the exterior flux becomes:

$$
\hat{\alpha}_{r}^{e}=\left|e_{r}\right|\left(\frac{\underline{f}\left(u_{K}\right)+\underline{f}\left(u_{K_{r}}\right)}{2} \cdot \underline{n}_{r}^{e}+\operatorname{sign}\left(\underline{a} \cdot \underline{n}_{r}^{e}\right) \frac{a_{r} \mid e_{r}}{2}\left(u_{K_{r}}-u_{K}\right)\right),
$$

for $r=i, j, k$.

\section{Remark 7}

In view of the linear case $\underline{f}(u)=\underline{a} u$, we can express $\underline{a}$ as linear combination of the local shape functions $\underline{p}_{r}$ of $R T_{0}(K)$. After some manipulations, we can exhibit the exterior numerical flux through the face $e_{r}$ :

$$
\hat{\alpha}_{r}^{e}=\frac{d_{r} u_{K}+d_{r^{\prime}} u_{K_{r}}}{d_{r}+d_{r^{\prime}}} a_{h_{r}}+\left.\operatorname{sign}\left(\underline{a} \cdot \underline{n}_{r}^{e}\right) \frac{\left|e_{r}\right| d_{r^{\prime}}}{d_{r}+d_{r^{\prime}}} a_{r}\right|_{r_{r}}\left(u_{K_{r}}-u_{K}\right)
$$

for $r=i, j, k$, and the approximation $a_{h}$ of $\underline{a}$ is defined by $\underline{a}_{h}=\sum_{r=i}^{k} a_{h_{r}} \underline{p}_{r}$.

\section{Remark 8}

To simplify, let $K$ be an equilateral triangle with centroid $G$, and let $K_{r}, r=i, j, k$ be the neighbouring equilateral triangles, with centroids $G_{r}(r=i, j, k)$. If we assume $\left.u\right|_{K}$ linear, and the least-squares gradient gradu(t) $\left.\right|_{K}=\left(a_{K}(t), b_{K}(t)\right)$ for the triangle $K$ is then chosen in order to minimize the functional

$$
F\left(a_{K}(t), b_{K}(t)\right)=\sum_{r=i}^{k}\left\{u_{K}(t)+\left.\overrightarrow{G G_{r}} \cdot \operatorname{gradu}(t)\right|_{K}-u_{K_{r}}(t)\right\}^{2} .
$$

Of course, the minimum is obtained when

$$
\frac{\partial F}{\partial a_{K}(t)}=\frac{\partial F}{\partial b_{K}(t)}=0
$$

for details and the expressions of $a_{K}(t)$ and $b_{K}(t)$ see (Champier, 1992).

Next, we define over all $K$

$$
P_{K}(x, y, t)=u_{G}(t)+\left(x-x_{G}\right) a_{K}(t)+\left(y-y_{G}\right) b_{K}(t),
$$

and the exterior and interior traces of $u$ at the face $e_{r}$ are now given by:

$$
u_{r}^{e}\left(t^{n}\right)=P_{K_{r}}\left(x_{m_{r}}, t^{n}\right) \quad \text { and } u_{r}^{i}\left(t^{n}\right)=P_{K}\left(x_{m_{r}}, t^{n}\right),
$$

where $x_{m_{r}}$ denote the coordinates of the center on sides $e_{r}$ (see Fig. 2). The resulting exterior flux is

$$
\begin{aligned}
\hat{\alpha}_{r}^{e}= & \left|e_{r}\right|\left(\frac{\underline{f}\left(u_{K}\right)+\underline{f}\left(u_{K_{r}}\right)}{2}\right) \cdot \underline{n}_{r}^{e} \\
& +\left.\operatorname{sign}\left(\underline{a} \cdot \underline{n}_{r}^{e}\right) \frac{\left|e_{r}\right|}{2} a_{r}\right|_{r}\left(u_{r}^{e}\left(t^{n}\right)-u_{r}^{i}\left(t^{n}\right)\right),
\end{aligned}
$$

for $r=i, j, k$. The cell average flux $\underline{f}\left(u_{K}\right),\left(\right.$ also $\left.f\left(u_{K_{r}}\right), \quad r=i, j, k\right)$ can be approximated by a second-order quadrature in time (such as the mid-point rule). The resulting scheme can be viewed as a natural new version of multidimensional extension of the second order central NT scheme (Nessyahu \& Tadmor, 1990). For other extensions of the NT scheme, see (Arminjon \& Viallon, 1995; Jiang \& Tadmor, 1998).

We close by noting that we can get a 2D extension of the family of semi-discrete central schemes of Kurganov and Tadmor (Kurganov \& Tadmor, 2000). Our recipe is similar to that above, taking into account the quadrature formula given below:

$$
\int_{K} \underline{f}\left(u(t) \underline{p}_{r} d x \simeq \frac{f\left(u_{r}^{i}\right)(t)}{2} \overrightarrow{A_{r} H_{K}} \quad r=i, j, k .\right.
$$


From this, we can express the exterior numerical flux in the form:

$$
\begin{aligned}
\hat{\alpha}_{r}^{e}= & \left|e_{r}\right|\left(\frac{\underline{f}\left(u_{r}^{i}(t)\right)+\underline{f}\left(u_{r}^{e}(t)\right)}{2}\right) \cdot \underline{n}_{r}^{e} \\
& +\left.\operatorname{sign}\left(\underline{a} \cdot \underline{n}_{r}^{e}\right) \frac{\left|e_{r}\right|}{2} a_{r}(t)\right|_{e_{r}}\left(u_{r}^{e}(t)-u_{r}^{i}(t)\right),
\end{aligned}
$$

for $r=i, j, k$, we then obtain the extension to the triangular grid case of the semi-discrete KT scheme (Kurganov \& Tadmor, 2000), and where $a_{r}(t)$ are the maximal local speeds. For more details see (Kurganov \& Tadmor, 2000).

\section{Discussion}

1- The extension to a system of equations can be carried out in the same fashion as (Fortin \& Serghini Mounim, 2005), and again with the aid of Roe's construction (ROE, 1981).

2- The generalization to a convection-diffusion equation can also be performed using the extension to 2D of the variational formulation used in (Fortin \& Serghini Mounim, 2005). We use, for this, the fact that the solutions to the general initial value problem of the viscous conservation laws converge, in the zero dissipation limit as $v$ goes zero, to the solutions of the hyperbolic conservation laws. Next, the "RH" jump conditions at the interelements are imposed again to the convective flux. Finally, we can establish the existence and uniqueness of the solution in this case taking the same approach to previous formulations, and using in particular an argument of regularity.

3- Note that the simplicity of the method makes it easy to extend to the three-dimensional case, in addition, if the triangulation of $\Omega$ is made of tetrahedrons, and the mesh is restricted to regular subdivisions of the domain, the quadrature formula given in Baranger et al (Baranger, Maitre, \& Oudin, 1996) can be used to diagonalize the mass matrix. Moreover, the result of (Baranger, Maitre, \& Oudin, 1996) cannot be extended to the general 3D case, for more details see (Thomas \& Trujillo, 1997).

4- Notice that the methods presented here can also be viewed as an extension of the mortar finite elements method to transport problems. On the other hand, we mention that there is a possible link between our methods and the mimetic finite difference methods (see for example (Hyman \& Shashkov, 1997)) that could be made by choosing an appropriate inner product.

Finally, the stability and the feasibility of the method had been successfully tested on numerical examples. For this, the dual mixed finite element method associated to the first-order accurate implicit Euler (for the time-discretization) has been experimented on structured and unstructured meshes see (Serghini Mounim, 2000).

\section{References}

Adams, R. A. (1975). Sobolev Spaces. Academic Press, New-York.

Agouzal, A., Baranger, J., Maitre, J. F., \& Oudin, F. (1995). Connection Between Finite volume and mixed finite element methods for a diffusion problem with nonconstant coefficients. Application to a convection diffusion problem. EastWest J. Num. Math., 3(4), 237-254.

Arminjon, P., \& Viallon, M. C. (1995). Généralisation du schéma de Nessyahu-Tadmor pour une équation hyperbolique à deux dimensions d'espace. Comptes Rendus de l'Acad. des Sciences, Paris, t.320, série I, 85-88.

Arnold, D. N., Brezzi, F. (1985). Mixed \& nonconforming finite element methods: implementation, postprocessing and error estimates. RAIRO Modél. Math. Anal. Numér., 19, 7-32.

Babuska, I. (1984). Error Bounds for Finite Elemnet Methods. Math., 16, 1-22.

Bank, R. E., \& Rose, D. J. (1987). Some error estimates for the box method. SIAM J. Num. An., 24, 777-787.

Baranger, J., Maitre, J. F., \& Oudin, F. (1993). Application de la théorie des éléments finis mixtes à l'étude d'une classe de schémas aux volumes finis pour les problèmes elliptiques. C. R. Acad. Sci. Paris, 316, série I, 509-512.

Baranger, J., Maitre, J. F., \& Oudin, F. (1996). Connection Between Finite Volume And Mixed Finite Element Methods. RAIRO $M^{2} A N, 30(4), 445-465$.

Beux, F., Lanteri, S., Dervieux, A., \& Larrouturou, B. (1993). Upwind Stabilization of Navier-Stokes Solvers. Rapport de Recherche, INRIA N 1885.

Brezzi, F. (1974). On the Existence, Uniqueness \& Approximation of Saddle-Point Problems Arising from Lagrangian Multipliers. RAIRO, Anal. Numer. Math., 2, 129-151.

Brezzi, F., Douglas, J., Duran, R., \& Fortin, M. (1987). Mixed finite elements for second order elliptic problems in three 
variables. Numer. Math., 51, 237-250,

Brezzi, F., Douglas, J., Fortin, M., Marini, L. D. (1987). Efficient rectangular mixed finite elements in two and three space variables. Math. Model. Numer. Anal., 21, 581-604.

Brezzi, F., Douglas, J., \& Marini, L. D. (1986). Recent results on mixed finite element methods for second order elliptic problems, in Vistas in Applied Math., Numerical Analysis, Atmospheric Sciences, Immunology (Balakrishanan, Dorodnitsyn, and Lions, eds.). Optimization Software Publications, New York.

Brezzi, F., \& Fortin, M. (1991). Mixed and Hybrid Finite Element Methods. Springer Series in Computational Mathematics. Springer-Verlag.

Brezzi, F., \& Marini, L. D. (1975). On the numerical solution of palte bending problems by hybrid methods. RAIRO, Anal., 5-50.

Champier, S. (1992). Convergence de schémas numériques type volumes finis pour la résolution d'équations hyperboliques. Thèse, Université de St-Etienne.

Ciarlet, P. G. (1978). The Finite Element Method for Elliptic Problems. North-Holland.

Ekeland, I., \& Temam, R. (1974). Analyse convexe et problèmes variationnels. Dunod, Paris.

Farhloul, M. (1991). Méthode d'éléments finis mixtes et volumes finis. $\mathrm{PhD}$ thesis, Université Laval.

Fortin, M., (1976). An analysis of the Convergence of Mixed Finite Element Methods. Rapport interne INRIA, 186.

Fortin, M. (1977). An analysis of the Convergence of Mixed Finite Element Methods. RAIRO, Anal. Numer., 11, 341-354.

Fortin, M., \& SERGHINI MOUNIM, A. (2005). Mixed and hybrid finite element methods for convection-diffusion equations and their relationships with finite volume, Part I. Calcolo, 42(1), 1-30.

Fraeijs de veubeke, B. X. (1965). Displacement and Equilibrium Models in the Finite Element Method. Stress Analysis (O.C. Zienkiewicz and G. Holister, eds.), Wiley, New-York.

Fraeijs de veubeke, B.X. (1975). Stress Function Approach. World Congress on the Finite Element Method in Structural Mechanics, Bournemouth.

Franca, L., \& Hugues, T. J. R. (1988). Two classes of mixed finite element methods. Comp. Meth. Appl. Mech. Eng., 69, $89-129$.

Girault, V., \& Raviart, P. A. (1980). Finite Elements Methods for Navier-Stokes Equations: Theory and Algorithms. Springer-Verlag.

Godlewski, E., \& Raviart, P. A. (1991). Hyperbolic Systems of Conservation Laws. Mathématiques et Applications. Ellipse.

Hansbo, P. (1994). Aspects of consevation in finite element flow computations. Comput. Meth. Appl. Mech. Engrg., 117, 423-437.

Hou, T. Y., \& Lefloch, P. (1992). Why conservative schemes converge to wrong solutions : Error analysis. Technical Report 255, Ecole Polytechique de Paris.

Hughes, T. J. R. (1978). A simple finite element scheme for developping upwind finite elements. Int. J. Num. Meth. Eng., $12,1359-1365$.

Hughes, T. J. R., \& BROOKS, A. (1979). A multidimensional upwind scheme with no crosswind diffusion. Finite Element Methods for convection dominated flows, AMD 34, ASME, New York.

Hyman, J. M., \& Shashkov, M. (1997). Natural discretizations for the divergence, gradient, and curl on logically rectangular grids. Int. J. Comp. Math. Appl., 33(4), 81-104.

Jiang, G.-S., \& Tadmor, E. (1998). Non-oscillatory central schemes for multidimensional hyperbolic conservation laws. SIAM J. Sci. Comput., 19, 1892-1917.

Kerkhoven, T., \& Jerome, J. (1990). $\mathrm{L}^{\infty}$ stability of finite element approximations to elliptic gradient equations. Numer. Math., 57, 561-575.

Kruzkov, S. N. (1970). First order quasilinear equtions in several independant variables. Numer. Math. USSR Sb., 10, 217-243.

Kurganov, A., \& levy, D. (2000). A Third-Order Semi-Discrete Central Scheme For Conservation Laws and Convection- 
Diffusion Equations. SIAM Journal on Scientific Computing, 22( 4), 1461-1488.

Kurganov, A., \& Tadmor, E., (2000). New high-resolution central schemes for nonlinear conservation laws and convectiondiffusion equations. J. Comput. Phys., 160 (1), 214-282.

Ladyzhenskaya, O. A., Solonnikov, V. A., \& Ouraltseva, N. N. (1967). Equations paraboliques linéaires et quasi linéaires. MIR.

Lazarov, R. D., Mishev, I. D., \& Vassilevski, P. S. (1996). Finite volume methods for convection-diffusion problems. SIAM J. Numer. Anal, 33, 31-55,

Lesaint, P., \& Raviart, P. A. (1974). On a finite element method for solving the neutron transport equation. In Mathematical aspect of finite elements in PDE.C de Boor ed. Academic Press, 89-123,

Leveque, R. J. (1990). Numerical Methods for Conservation Laws. Lectures in Mathematics. Birkhauser.

Leveque, R. J. (2002). Finite Volume Methods for Hyperbolic Problems. Cambridge Texts In Applied Mathematics.

Majda, A. (1984). Compressible Fluid Flow and Systems of Conservation Laws in Serval Space Variables. Bull. Applied Math. Sciences, 53. Springer-Verlag,

Mishev, I. D. (1998). Finite Volume Methods on Voronoi Meshes. Numer. Meth. PDE, 14,(2), 193-212,

Nedelec, J. C. (1986). A New Family of Mixed Finite Elements in $\mathrm{R}^{3}$. Numer. Math., 50, 57-81.

Nessyahu, H., \& Tadmor, E. (1990). Non-oscillatoy Central Differencing for Hyperbolic Conservation Laws. JCP, 87(2), 408-463.

Raviart, P. A., \& Thomas, J. M. (1977). A Mixed Finite Element Method for 2-nd Order Elliptic Problems. Lecture Notes in Mathematics, 606, Springer-Verlag, New-York, 292-315.

Roe, P. L. (1985). Some contributions to the modeling of discontinuous flows. Lect. Notes Appl. Math., 22, $163-193$.

Roe, P. L. (1981). Approximate Riemann solvers, parameter vectors, and difference schemes. J. Comput. Phys., 43, 357-372.

Sacco, R., \& Saleri, F. (1997). Mixed Finite Volume Methods For Semiconductor Device Simulation. Numer. Methods Partial Differential Equations, 13, 215-236.

Serghini Mounim, A. (2000). Méthode d'éléments finis mixtes: Application aux équations de convection-diffusion et Navier-Stokes. Ph.D. thesis, Université Laval.

Smoller, J. (1994). Shock Waves and Reaction-Diffusion Equations. Spriger-Verlag, 2 edition.

Thomas, J. M. (1977). Sur l'analyse numérique des méthodes d'éléments finis hybrides et mixtes. Thèse, Université Pierre et Marie Curie, Paris 6.

Thomas, J. M., \& Trujillo, D. (1997). Finite volume methods for elliptic problems; Convergence on unstructured meshes. In. Numerical Methods in Mechanics, Conca C, Gatica G(eds). Pitman Research Notes in Mathematics Series, 371. Addison-Wesley Longman: Reading MA, 163-174.

\section{Copyrights}

Copyright for this article is retained by the author(s), with first publication rights granted to the journal.

This is an open-access article distributed under the terms and conditions of the Creative Commons Attribution license (http://creativecommons.org/licenses/by/4.0/). 